\title{
$-196-$
}

A insuficiencia dos que foram incumbidos de missão tão ardua, e a escassez do tempo que lhes foi dado, justificão de sobra, aos olhos dos doutos, a grande deficiencia deste trabalho: a commissão, porém, tem a consciencia de que procurou cumprir o seu dever.

Rio de Janeiro, sala da commissão, na Secretaria da Justiça e Negocios Interiores, 27 de Julho de 1893. - Antonio José Rodrigues Torres, neto, presidente. - Antonio Dino da C. Bueno, relator.-M. do N. Machado Portella Junior.

\section{PRIMEIRO ANNO DO CURSO JURIDICO, EM 1828}

Para aqui trasladamos (I), como subsidio á historia da Faculdade de Direito de S. Paulo a lista dos estudantes matriculados em $\mathrm{r} 828$, os primeiros que iniciaram o seu curso nesta Faculdade.

I Antonio Paes de Camargo, natural da villa de Sorocaba, provincia de S. Paulo.

2 Manuel Dias de Toledo, natural da villa de Porto Feliz, provincia de S. Paulo.

3 José Antonio Pimenta Bueno, natural desta cidade de $\mathrm{S}$. Paulo.

4 José Christiano Garção Stockler, natural da villa de S. João d'El-rei, provincia de Minas Geraes.

5. Vicente Pires da Motta; natural desta cidade de S. Paulo.

6. José Fernando de Almeida, natural da cidade do Rio de Janeiro.

7. Francisco José Pinheiro Guimarães, natural da cidade do Rio de Janeiro.

8. José Ignacio Vaz Vieira, natural da cidade do Rio de Janeiro.

(1) O Farol Paulistano, n. 104, de 16 de Abril de 1828 . 


\section{$-197-$}

9. Marcellino Ferreira Bueno, natural desta cidade de S. Paulo.

Io. Felisberto Gomes Jardim, natural da villa de Sorocaba, provincia de S. Paulo.

I I. João José Coutinho, natural da cidade do Rio de Janeiro.

I 2. Pedro Antonio de Oliveira, natural da cidade do Rio de Janeiro.

I3. Manuel de Jesus Valdetaro, natural da cidade do Rio de Janeiro.

14. Emilio Paulo de Carvalho, natural desta cidade de S. Paulo.

I 5. José Gaspar dos Santos Lima, natural da villa Nova do Principe, provincia de S. Paulo.

16. Fernando Sebastiāo Dias da Motta, natural da cidade do Rio de Janeiro.

I7. José Barbosa de Oliveira, natural da cidade da Bahia.

18. Antonio Mariano de Azevedo Marques, natural desta cidade de S. Paulo.

I 9. Manuel Alves Alvim, natural desta cidade de S. Paulo.

20. José Antonio dos Reis, natural desta cidade de S. Paulo.

21. Manuel Joaquim do Amaral Gurgel, natural desta cidade de S. Paulo.

22. João Affonso de Moraes Torres, natural da cidade do Rio de Janeiro.

23. Francisco de Assis Pupo, natural da villa de Iguape, provincia de S. Paulo.

24. Joaquim Firmino Pereira Jorge, natural da villa de S. Sebastião, provincia de S. Paulo.

25. Tristão Antonio de Alvarenga, natural da villa da Campanha da Princeza, provincia de Minas Geraes. 


\section{$-198-$}

26. Francisco Augusto de Oliveira Muniz, natural da villa de Iguape, provincia de S. Paulo.

27. Francisco Antonio da Costa Machado, natural da villa de Iguape, provincia de S. Paulo.

28. Joaquim Pedro da Costa Lobo, natural da cidade da Bahia.

29. Luiz Fortunato de Brito, natural da cidade do Rio de Janeiro.

3o. João José Cardoso, natural da cidade do Rio de Janeiro.

3 I. Manuel Euphrasio de Azevedo Marques, natural desta cidade de S. Paulo.

32. Cyrino Antonio de Lemos, natural do $S$. Gonçalo da Campanha, provincia de Minas Geraes.

33. Jeronymo Maximo de Oliveira e Castro, natural da cidade de Ouro Preto, provincia de Minas Geraes.

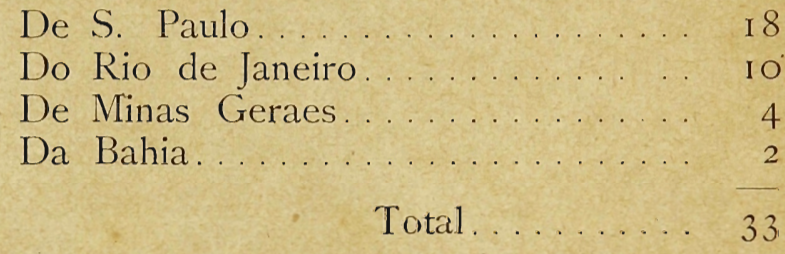

